\title{
Ursolic acid alleviates inflammation and against diabetes-induced nephropathy through TLR4-mediated inflammatory pathway
}

\author{
JIAN LI $^{1 *}$, NAN LI ${ }^{1 *}$, SHUANGTONG YAN ${ }^{1}$, MINYAN LIU ${ }^{1}$, \\ BANRUO SUN $^{1}$, YANHUI LU ${ }^{1}$ and YINGHONG SHAO ${ }^{2}$ \\ ${ }^{1}$ Department of Geriatric Endocrinology, Chinese PLA General Hospital, National Clinical Center of Geriatric Medicine; \\ ${ }^{2}$ Outpatient Department, Chinese PLA General Hospital, Beijing 100853, P.R. China
}

Received March 19, 2018; Accepted June 25, 2018

DOI: $10.3892 / \mathrm{mmr} .2018 .9429$

\begin{abstract}
Ursolic acid (UA) is a triterpenoid isolated from Chinese herbal medicine. It is extensively distributed in the plant kingdom in at least 63 Chinese herbal medicines of 26 families. UA has multiple bioactivities, including anti-viral hepatitis, antitumor, anti-oxidation, anti-bacterium and anti-inflammation. The aim of this in vitro study was to examine the effects of UA on diabetes-induced nephropathy and its possible mechanism. In mice with diabetes-induced nephropathy, UA increased the body weight, reduced kidney/ body weight index, protected kidney cells, alleviated inflammation [tumor necrosis factor (TNF)- $\alpha$, interleukin (IL)-1 $\beta$, IL- 6 and IL-18 levels] and kidney cell damage. It was also indicated that UA suppressed Toll-like receptor 4 (TLR4), myeloid differentiation factor 88 and nuclear factor- $\kappa \mathrm{B}$ protein expression in mice with diabetes-induced nephropathy. The inhibition of TLR4 increased the anti-inflammation of UA on inflammation in rat with diabetes-induced nephropathy through the TLR4 signaling pathway. In conclusion, UA alleviates inflammation and inhibits diabetes-induced nephropathy through a TLR4-mediated inflammatory pathway. The present findings indicated that UA may be a possible therapeutic agent against diabetic nephropathy.
\end{abstract}

\section{Introduction}

Diabetes mellitus (DM) is a chronic non-infectious disease. Of them, disease resulted from islet B cell damage is called type 1 DM (T1DM), while disease in which the body can produce insulin but the cells can not utilize the insulin is called type 2

Correspondence to: Dr Yinghong Shao, Outpatient Department, Chinese PLA General Hospital, 28 Fuxing Road, Beijing 100853, P.R. China

E-mail: shaoyh021206@sina.com

\section{${ }^{*}$ Contributed equally}

Key words: ursolic acid, diabetic nephropathy, inflammation, TLR4, MyD88, NF-кB
DM (T2DM) (1). DM morbidity shows an increasing trend globally with the improvement in people's living standard and changes in lifestyle (2). It is conservatively estimated that, the global DM cases would increase from 0.382 billion in 2013 to 0.592 billion in 2035 (3). Large epidemiological survey in China discovers that, DM morbidity in people aged over 20 years is $9.7 \%$. Of them, T2DM accounts for $90 \%$, and patients combined with diabetic nephropathy (DN) have taken up 20-40\% (3). DN is a common chronic complication of DM, which manifests as proteinuria and hypertension. Typically, it is characteristic of the sign of urinary albumin excretion rate (4). Meanwhile, it is also the most critical cause of end-stage renal disease (ESRD) (4). The growing ESRD cases have caused tremendous economic burdens on the country (5). Research also reports that, DN-induced deaths account for $60 \%$ of the total DM-related deaths (5).

The pathogenesis of $\mathrm{DN}$ is mainly explained from genetic susceptibility factor, abnormal glucose metabolism pathway, kidney hemodynamic changes, inflammatory response theory and cytokine theory (6). An increasing number of scholars accept the inflammatory response theory (7). In other words, $\mathrm{DM}$ is a natural focal disease, with inflammatory response in its course (8). It is an inflammatory disease induced by metabolic disorder (7). In the genesis and development of microvascular complications such as DN, inflammatory response also plays a vital role. Similarly, this view is verified in related clinical and laboratory research (6). However, the genesis of inflammatory response is complicated. It is a disease resistance response occurring in systemic tissues and multiple organs (7). Meanwhile, it is accompanying with body fever and leukocytosis (6). In essence, it is a process in which inflammatory factors fight against the body (6).

Existing studies suggest that, Toll-like receptor 4 (TLR4) is a major receptor of the natural immune system to recognize pathogenic microorganism (9). The TLR4 signaling pathway is activated upon the stimulation of lipopolysaccharide (LPS). As a result, the lipid of LPS A binds with LPS-binding protein (LBP) outside the relevant cells to form the LPS-LBP complex. Such complex binds with CD14 on cell membrane surface, thus activating TLR4. The activated TLR4 binds with the homodomain of myeloid differentiation factor 88 (MyD88) through the internal segment of its cytoplasma (10). In addition, the death domain (DD) of MyD88 can recruit 
the downstream molecules containing DD (11). Therefore, it can induce the release of pro-inflammatory cytokines tumor necrosis factor (TNF)- $\alpha$, interleukin (IL)-1 and IL-6, thus exerting the immune response effect (11).

Ursolic acid (UA) is the anti-hepatitis active ingredient extracted from the Caprifoliaceae plant Sambucus chinensis. It is a pentacyclic triterpenoid extensively distributed in the nature (12). Typically, it exists in the free form or binds with sugar to form glycoside in plants like Sambucus chinensis, Fructus crataegus, Arbutus menziesii, Prunella vulgaris, glossy privet fruit, plantain herb, Incarvillea arguta, and oldenlandia diffusa $(13,14)$. It has multiple clinical pharmacological effects, including antitumor, anti-hepatitis, anti-inflammation and anti-bacterium. It is the ideal drug to treat viral hepatitis, with low toxicity and little side effects (13). The aim of this in vitro study is to examine the effects of UA alleviates diabetes-induced nephropathy and its possible mechanism.

\section{Materials and methods}

Animals and experimental rat model. Adult male Wistar rats (200-230 g) were obtained from Beijing Vital River Laboratory Animal Technology Co., Ltd. (Beijing, China) and were housed in standard environmental conditions maintained at $22 \pm 2{ }^{\circ} \mathrm{C}, 55-60 \%$ humidness, freely access to food and water with $12 \mathrm{~h}$ light-dark cycle. All rats were randomly divided into three groups: i) Sham + vehicle group $(n=6)$; ii) Nephropathy + vehicle group $(n=6)$; and iii) Nephropathy $+25 \mathrm{mg} / \mathrm{kg}$ UA group $(\mathrm{n}=6)$. All rats of Nephropathy + vehicle group or Nephropathy $+25 \mathrm{mg} / \mathrm{kg}$ UA group were received $50 \mathrm{mg} / \mathrm{kg}$ of intra-peritoneal injection of streptozocin for 60 days. All rats of Nephropathy $+25 \mathrm{mg} /$ $\mathrm{kg}$ UA group were gavaged with $25 \mathrm{mg} / \mathrm{kg}$ UA for 60 days. This study was approved by the Ethics Committee of Chinese PLA General Hospital. At the end of these experiments, all animals were anesthetized by $35 \mathrm{mg} / \mathrm{kg}$ pentobarbital sodium and sacrificed using decollation.

Hematoxylin and eosin $(H \& E)$ staining. After treatment with UA, kidney tissue samples were collected and fixed with $4 \%$ paraformaldehyde for $24 \mathrm{~h}$. Kidney tissue samples were dehydrated, embedded, and sliced, and cut into $5 \mu \mathrm{m}$ thickness. Sections of $5 \mu \mathrm{m}$ thickness were stained with H\&E sassy for $5 \mathrm{~min}$ and then examined using transmission electron microscopy (H7650).

ELISA kits for inflammation. After treatment with UA, peripheral blood or cells were collected and centrifuged at $1,000 \mathrm{x} \mathrm{g}$ for $5 \mathrm{~min}$ at $4^{\circ} \mathrm{C}$. TNF- $\alpha$, IL-1 $\beta$, IL- 6 and IL-18 levels were measured using ELISA kits.

Western blot analysis. After treatment with UA, kidney tissue samples or cells were collected and homogenated using RIPA assay for $30 \mathrm{~min}$ at $4^{\circ} \mathrm{C}$. Proteins were collected, electrophoresed via $10 \%$ SDS-PAGE, and transferred to polyvinylidene fluoride membranes. The membranes were blocked with $5 \%$ non-milk in TBST for $1 \mathrm{~h}$ at $37^{\circ} \mathrm{C}$ and incubated at $4^{\circ} \mathrm{C}$ overnight with the following primary antibodies: TLR4, MyD88, NF- $\kappa$ B and GAPDH (Santa Cruz Biotechnology, Inc., Dallas, TX, USA). The membranes were washed with TBST for $15 \mathrm{~min}$ and incubated with species-specific horseradish peroxidase-conjugated secondary antibodies (Santa Cruz Biotechnology, Inc.) for $1 \mathrm{~h}$ at $37^{\circ} \mathrm{C}$. Protein bands were detected using a Western Bright Enhanced Chemiluminescence detection system (Advansta, Inc., Menlo Park, CA, USA).

Statistical analysis. Values were expressed as mean \pm SEM. One-way ANOVA was followed by Tukey's post hoc multiple comparison test for statistical analysis.

\section{Results}

UA prevented diabetes-induced nephropathy in rat. First, we explored whether the effect of UA prevented diabetes-induced nephropathy. The structural formula of UA was showed at Fig. 1A. As observed in Fig. 1B, glomerulus appeared damage in rat with diabetes-induced nephropathy, compared with sham group. As showed in Fig. 1C and D, body weight was inhibited, blood glucose levels were increased in rat with diabetes-induced nephropathy, compared with sham group. Then, treatment with UA prevented glomerular damage, increased body weight and reduced blood glucose levels in rat with diabetes-induced nephropathy, compared with sham group (Fig. 1B-D).

UA protected kidney cell in rat with diabetes-induced nephropathy. Kidney weight/body weight ratio was decreased, blood urea nitrogen (BUN) and Creatinine in serum were promoted, and Albumin in serum was increased in serum of rat with diabetes-induced nephropathy, compared with sham group (Fig. 2). These indexes were reversed by UA in rat with diabetes-induced nephropathy, compared with diabetes-induced nephropathy model group (Fig. 2).

UA prevented inflammation in rat with diabetes-induced nephropathy through TLR4 pathway. Diabetic rats showed a significant elevation of TNF- $\alpha$, IL-1 $\beta$, IL- 6 and IL-18 levels in rat with diabetes-induced nephropathy, compared with sham group (Fig. 3). Treatment with UA reduced TNF- $\alpha$, IL-1 $\beta$, IL-6 and IL-18 levels in rat with diabetes-induced nephropathy, compared with diabetes-induced nephropathy model group (Fig. 3). Diabetic rats showed increase of protein expression levels of TLR4, MyD88 and NF- $\mathrm{KB}$ in rat with diabetes-induced nephropathy, compared with sham group (Fig. 4). Treatment with UA suppressed TLR4, MyD88 and $\mathrm{NF}-\kappa \mathrm{B}$ protein expression in rat with diabetes-induced nephropathy, compared with diabetes-induced nephropathy model group (Fig. 4).

The inhibition of TLR4 increased the anti-inflammation of UA on inflammation in rat with diabetes-induced nephropathy through TLR4 pathway. To assess the role of TLR4 in the anti-inflammation of UA on inflammation in vitro model, TLR4 inhibitor (TAK-242) suppressed TLR4 protein expression in vitro model by UA. As showed in Fig. 5, TLR4 inhibitor suppressed TLR4, MyD88 and NF- $\kappa \mathrm{B}$ protein expression in rat with diabetes-induced nephropathy by UA, compared with only treatment with UA group. However, TLR4 inhibitor promoted TNF- $\alpha$, IL-1 $\beta$, IL- 6 and IL-18 levels in rat with diabetes-induced nephropathy by UA, compared with only treatment with UA 
A

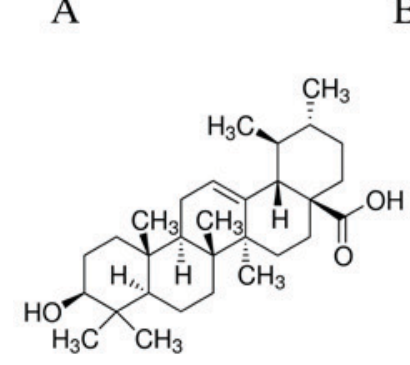

B
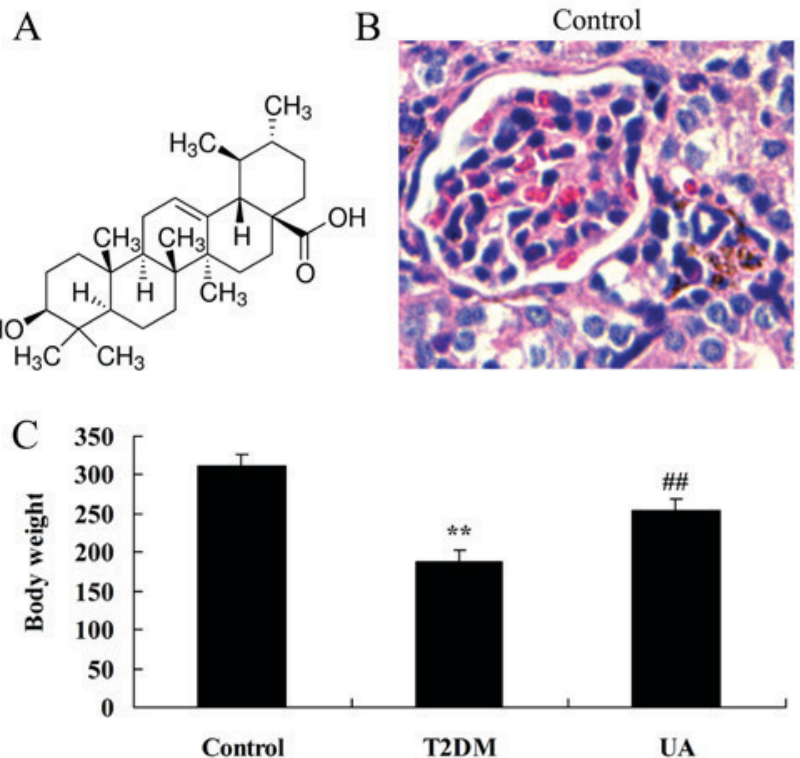

$\mathrm{D}$

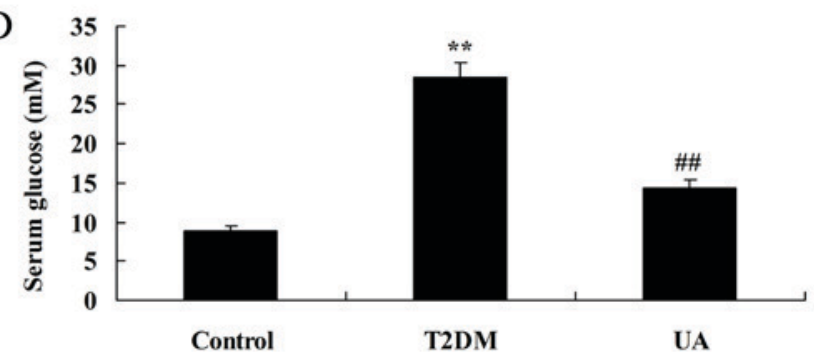

Figure 1. UA prevented diabetes-induced nephropathy in rats. (A) The structural formula of UA, (B) H\&E staining in the glomerulus (magnification, $\mathrm{x} 400$ ), (C) body weight and (D) blood glucose levels were indicated. ${ }^{* *} \mathrm{P}<0.01$ compared with control rat group, ${ }^{\# \#} \mathrm{P}<0.01$ compared with diabetes-induced nephropathy group. Control, control rat group; T2DM, diabetes-induced nephropathy in rat model group; UA, treatment with UA in rat of diabetes-induced nephropathy group. UA, ursolic acid; H\&E, hematoxylin and eosin; T2DM, type 2 diabetes mellitus.

A

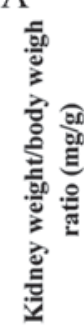

$\mathrm{C}$

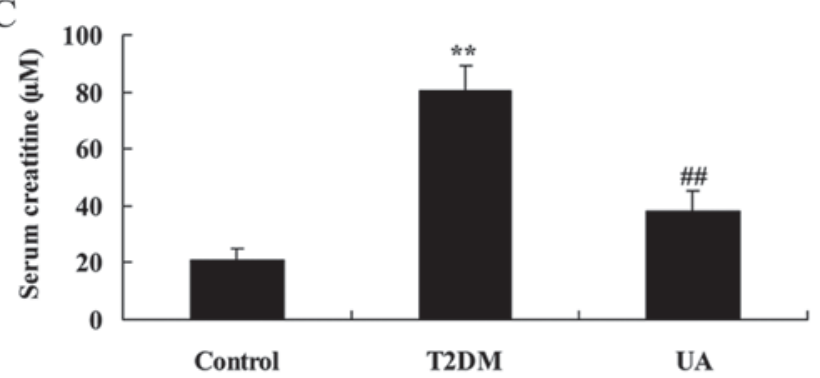

B

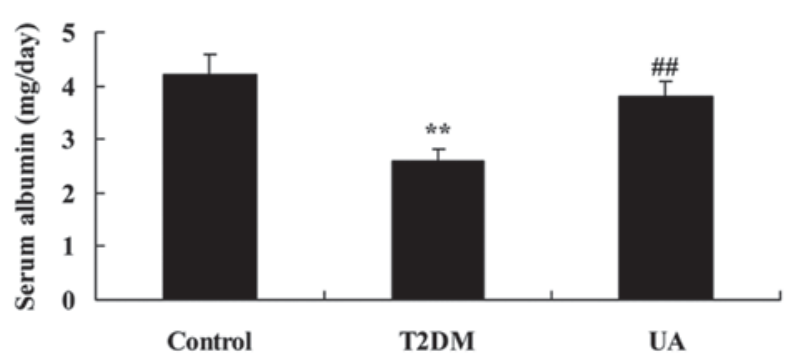

$\mathrm{D}$

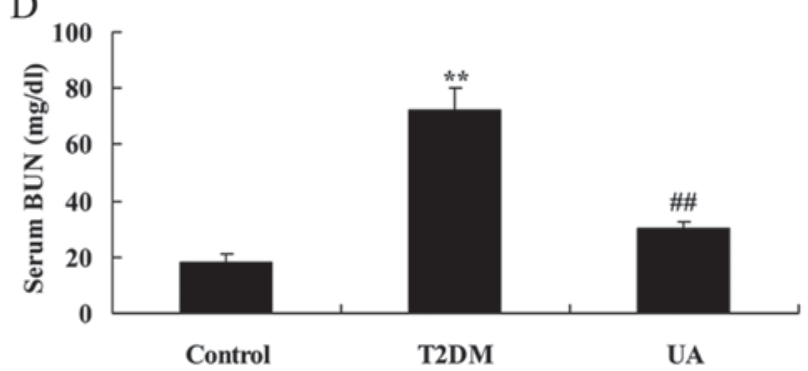

Figure 2. UA protected kidney cell in rat with diabetes-induced nephropathy. (A) Kidney weight/body weight ratio, (B) Albumin, (C) BUN and (D) Creatinine levels. Control, control rat group; T2DM, diabetes-induced nephropathy in rat model group; UA, treatment with UA in rat of diabetes-induced nephropathy group. ${ }^{* *} \mathrm{P}<0.01$ compared with control rat group, ${ }^{\# \#} \mathrm{P}<0.01$ compared with diabetes-induced nephropathy group. UA, ursolic acid; BUN, blood urea nitrogen; T2DM, type 2 diabetes mellitus.

group (Fig. 6). TLR4 inhibitor reduced glomerulus appeared damage, kidney weight/body weight ratio, BUN and Creatinine in serum, albumin and protein of urine, and increased albumin in serum of rat with diabetes-induced nephropathy by UA, compared with only treatment with UA group (Fig. 7).

\section{Discussion}

DN morbidity is growing in numerous countries in the world with the improvement in people's living standard (1). DN is one of the severe complications of DM-related microvascular lesion. It is also one of the major causes leading to ESRD. The pathogenesis of DN is complicated, which is once considered to be a metabolic disorder (15). Existing studies suggest that, DN genesis is closely related to genetic, immune and environmental factors (15). These findings suggest that treatment with UA prevented glomerular damage, increased body weight and Albumin, reduced blood glucose levels, BUN, Creatinine in serum levels and kidney weight/body weight ratio in rat with diabetes-induced nephropathy. 

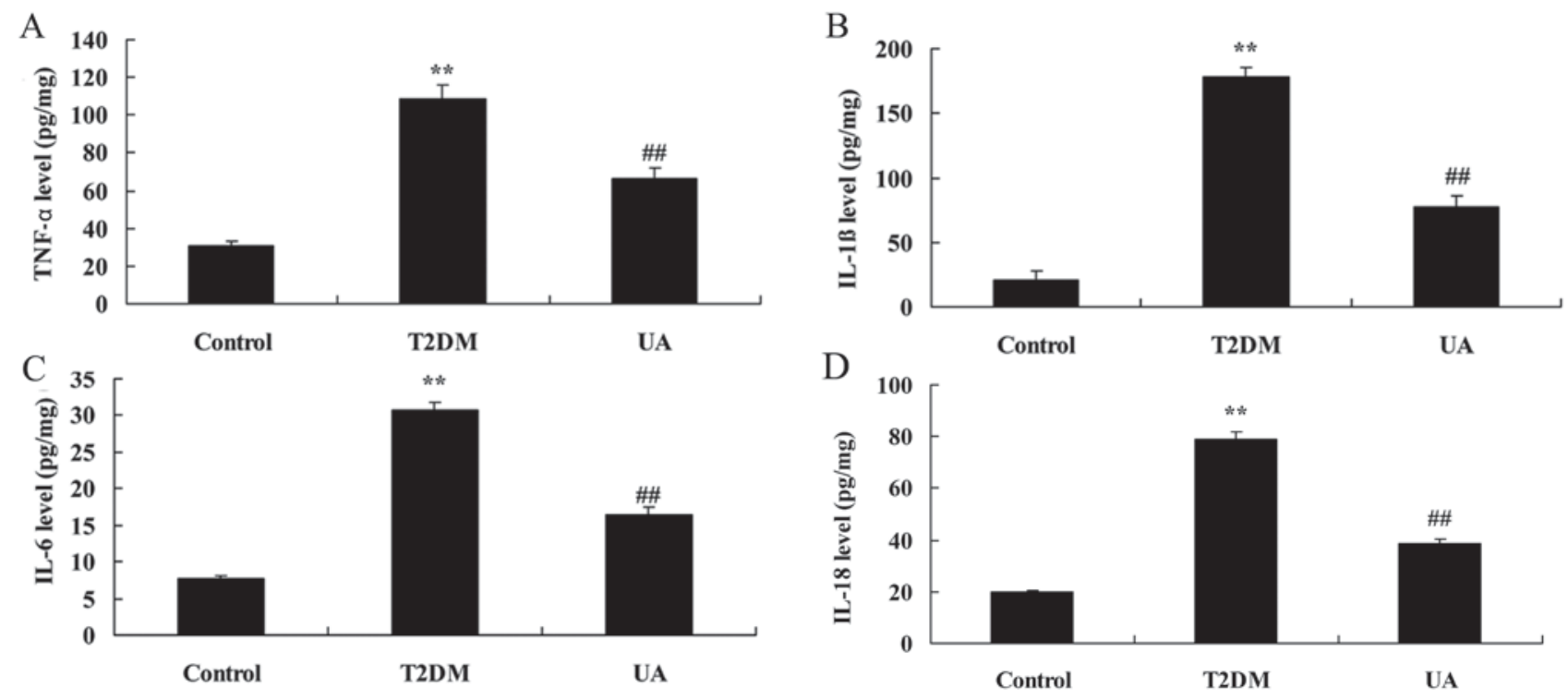

Figure 3. UA prevented inflammation in rat with diabetes-induced nephropathy through TLR4 pathway. (A) TNF- $\alpha$, (B) IL-1 $1 \beta$, (C) IL-6 and (D) IL-18 levels ${ }^{* *} \mathrm{P}<0.01$ compared with control rat group, ${ }^{\# \#} \mathrm{P}<0.01$ compared with diabetes-induced nephropathy group. Control, control rat group; T2DM, diabetes-induced nephropathy in rat model group; UA, treatment with UA in rat of diabetes-induced nephropathy group. UA, ursolic acid; TLR4, Toll-like receptor 4; T2DM, type 2 diabetes mellitus; TNF, tumor necrosis factor; IL, interleukin.
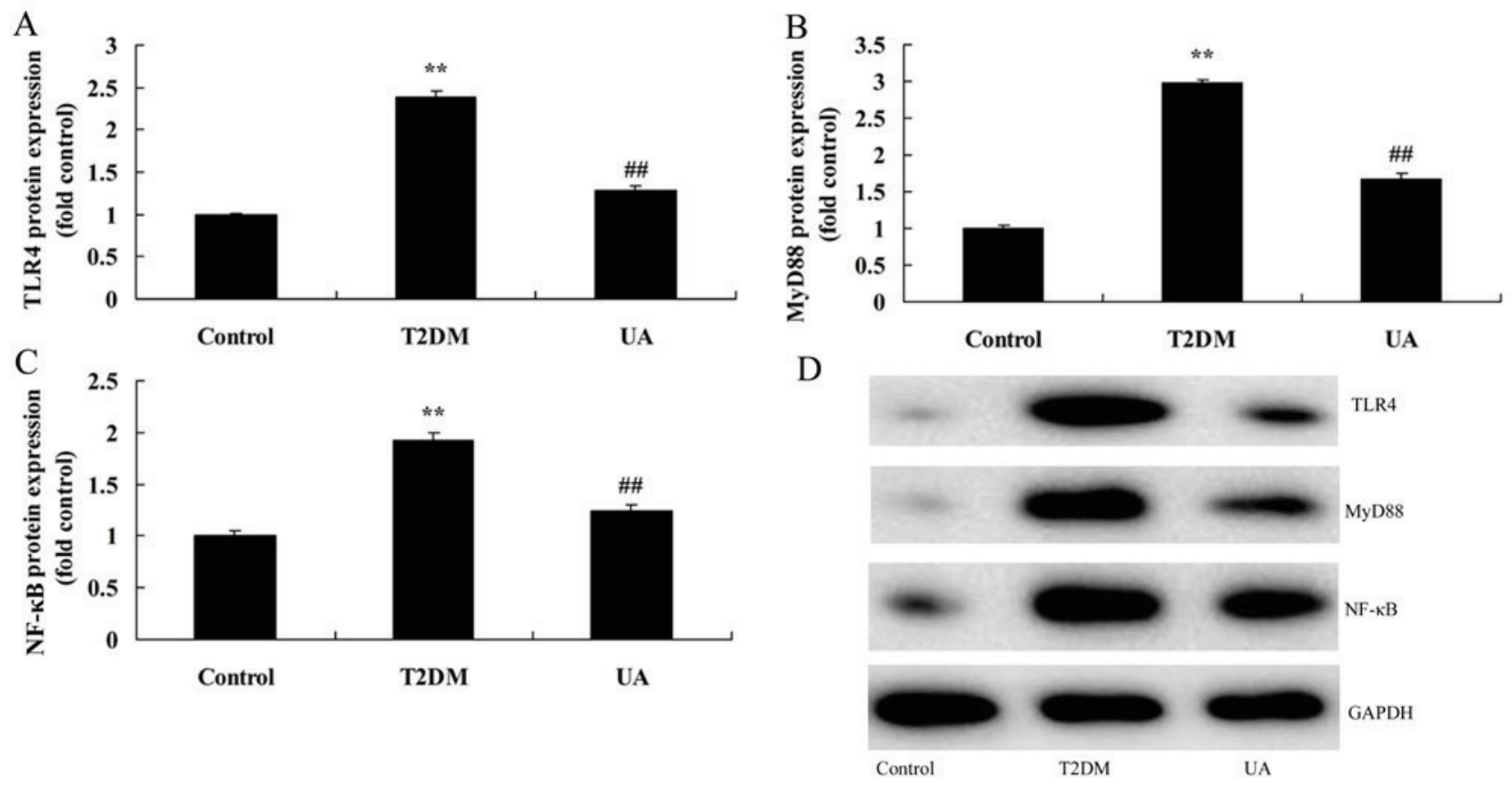

Figure 4. UA suppressed TLR4 pathway in rat with diabetes-induced nephropathy through TLR4 pathway. (A-C) TLR4, MyD88 and NF- $\mathrm{B}$ protein expression by statistical analysis and (D) western blotting assays. ${ }^{* *} \mathrm{P}<0.01$ compared with control rat group, ${ }^{\# \#} \mathrm{P}<0.01$ compared with diabetes-induced nephropathy group. Control, control rat group; T2DM, diabetes-induced nephropathy in rat model group; UA, treatment with UA in rat of diabetes-induced nephropathy group. UA,

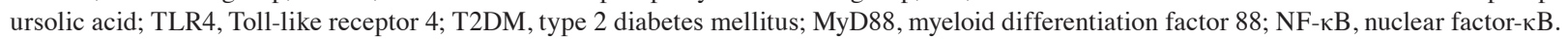

The pathogenesis of DN is complex, which is previously considered to be related to mechanisms such as gene polymorphism, abnormal kidney hemodynamics, abnormal growth factor metabolism and oxidative stress (16). In recent years, an increasing number of studies find that the genesis and development of DN is closely related to inflammation (16). Research also discovers that, metabolic disorders like hyperglycemia will damage the renal cells, and promote them to secrete and release the inflammatory factors (8). In this way, they will induce the cascade reaction and induce renal damage (17). The immunity and inflammation theories have received wide attention in recent years. It is suggested that endothelial injury and platelet activation are closely correlated with the genesis and development of DM (17). Wang et al showed that UA ameliorates oxidative stress, inflammation and fibrosis in diabetic cardiomyopathy rats (18). We found that UA prevented TNF- $\alpha$, IL-1 $\beta$, IL- 6 and IL-18 levels in rat with diabetes-induced nephropathy. 

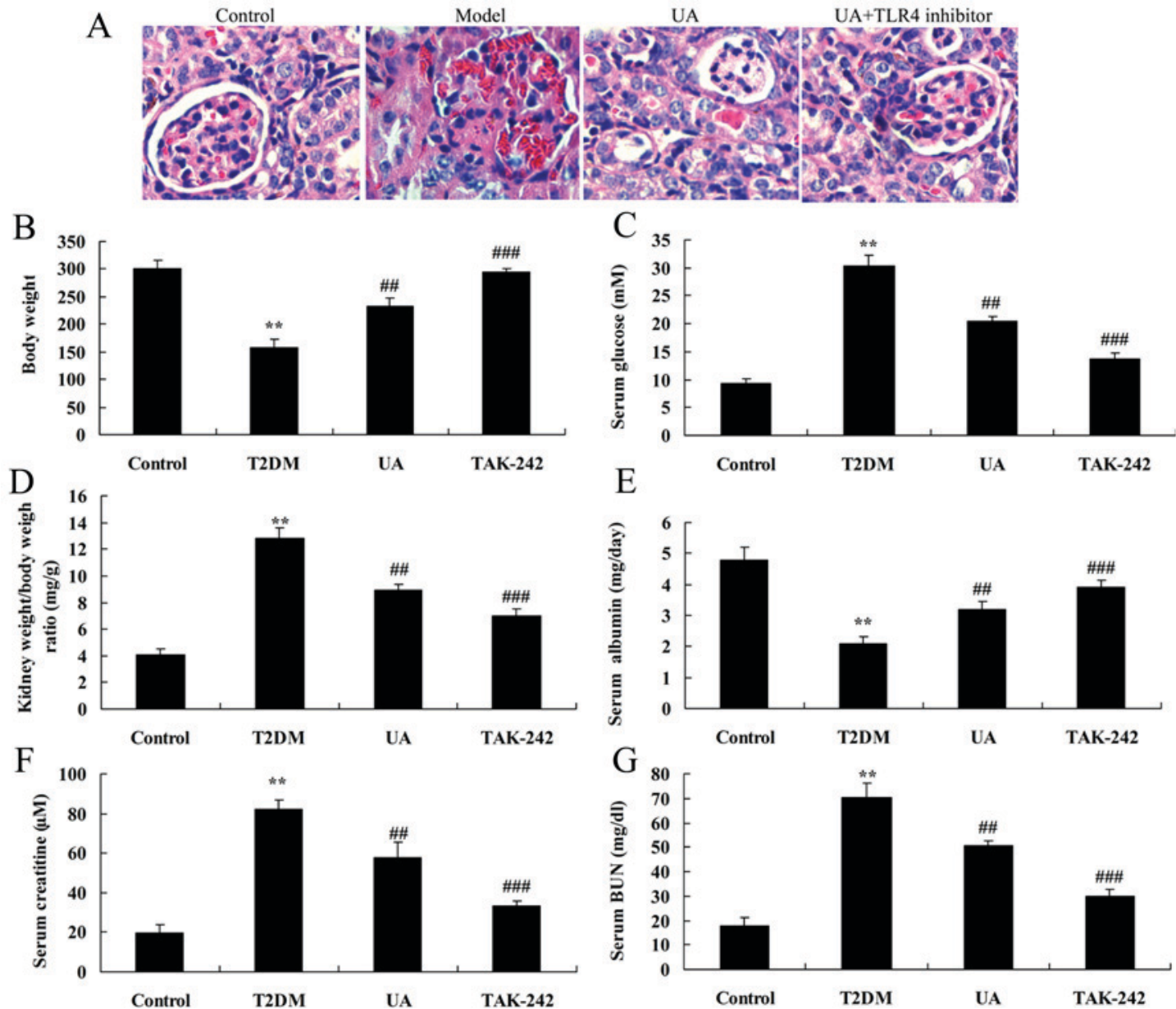

Figure 5. Inhibition of TLR4 increased the anti-inflammation of UA on nephropathy in rat with diabetes-induced nephropathy through TLR4 pathway. (A) H\&E staining in the glomerulus (magnification, $\mathrm{x} 400$ ), (B) body weight, (C) blood glucose levels, (D) kidney weight/body weight ratio, (E) albumin, (F) BUN and $(\mathrm{G})$ creatinine levels were indicated. ${ }^{* *} \mathrm{P}<0.01$ compared with control rat group ${ }^{\# \#} \mathrm{P}<0.01$ compared with diabetes-induced nephropathy group,

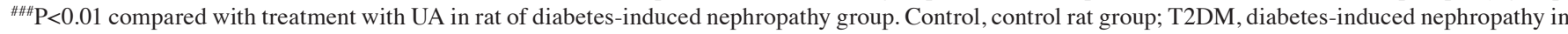
rat model group; UA, treatment with UA in rat of diabetes-induced nephropathy group; TAK-242, treatment with UA and TAK-242 in rat of diabetes-induced nephropathy group. UA, ursolic acid; TLR4, Toll-like receptor 4; T2DM, type 2 diabetes mellitus; BUN, blood urea nitrogen.
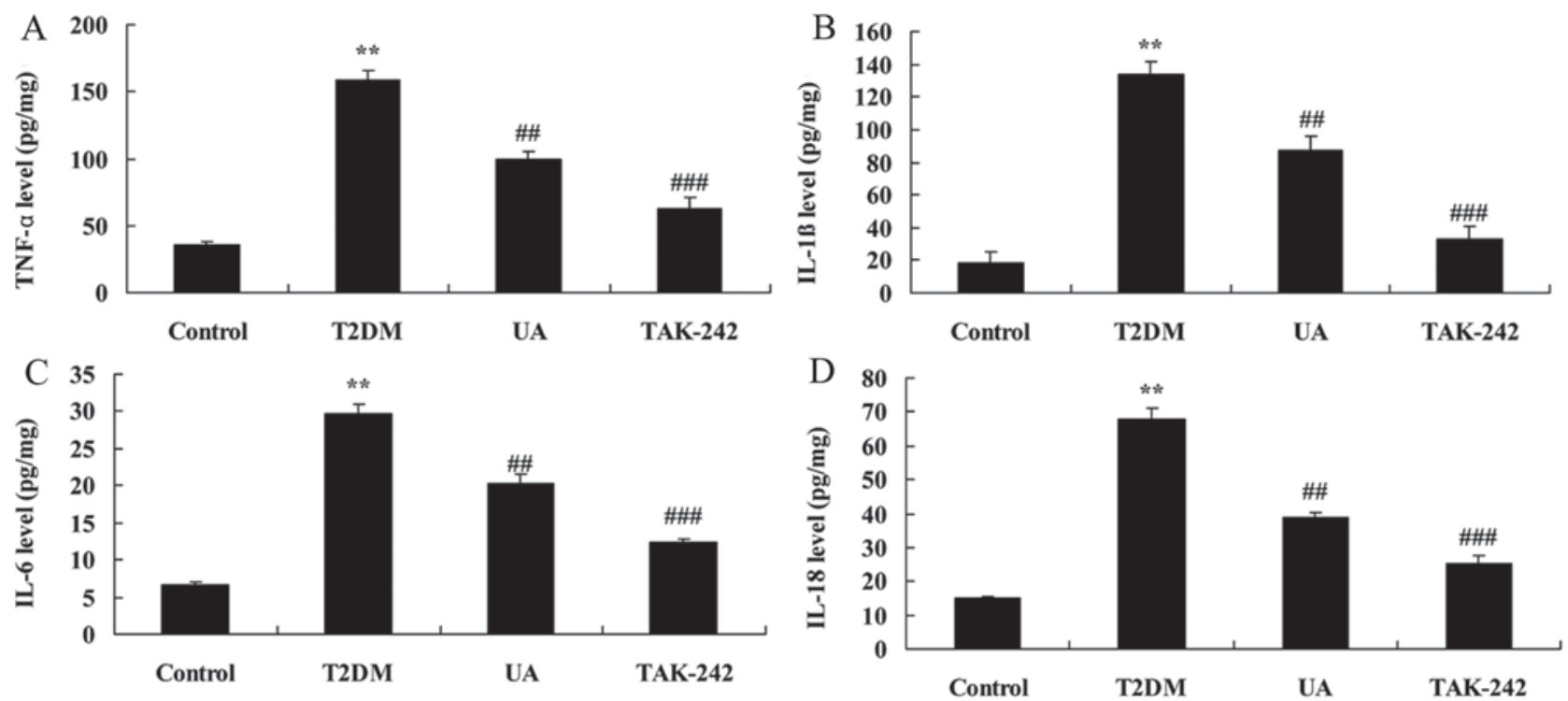

Figure 6. Inhibition of TLR4 increased the anti-inflammation of UA on inflammation in rat with diabetes-induced nephropathy. (A) TNF- $\alpha$, (B) IL-1 $\beta$, (C) IL-6 and (D) IL-18 levels. ${ }^{* *} \mathrm{P}<0.01$ compared with control rat group, ${ }^{\# \#} \mathrm{P}<0.01$ compared with diabetes-induced nephropathy group, ${ }^{\# \# \#} \mathrm{P}<0.01$ compared with treatment with UA in rat of diabetes-induced nephropathy group. Control, control rat group; T2DM, diabetes-induced nephropathy in rat model group; UA, treatment with UA in rat of diabetes-induced nephropathy group; TAK-242, treatment with UA and TAK-242 in rat of diabetes-induced nephropathy group. UA, ursolic acid; TLR4, Toll-like receptor 4; T2DM, type 2 diabetes mellitus; TNF, tumor necrosis factor; IL, interleukin. 

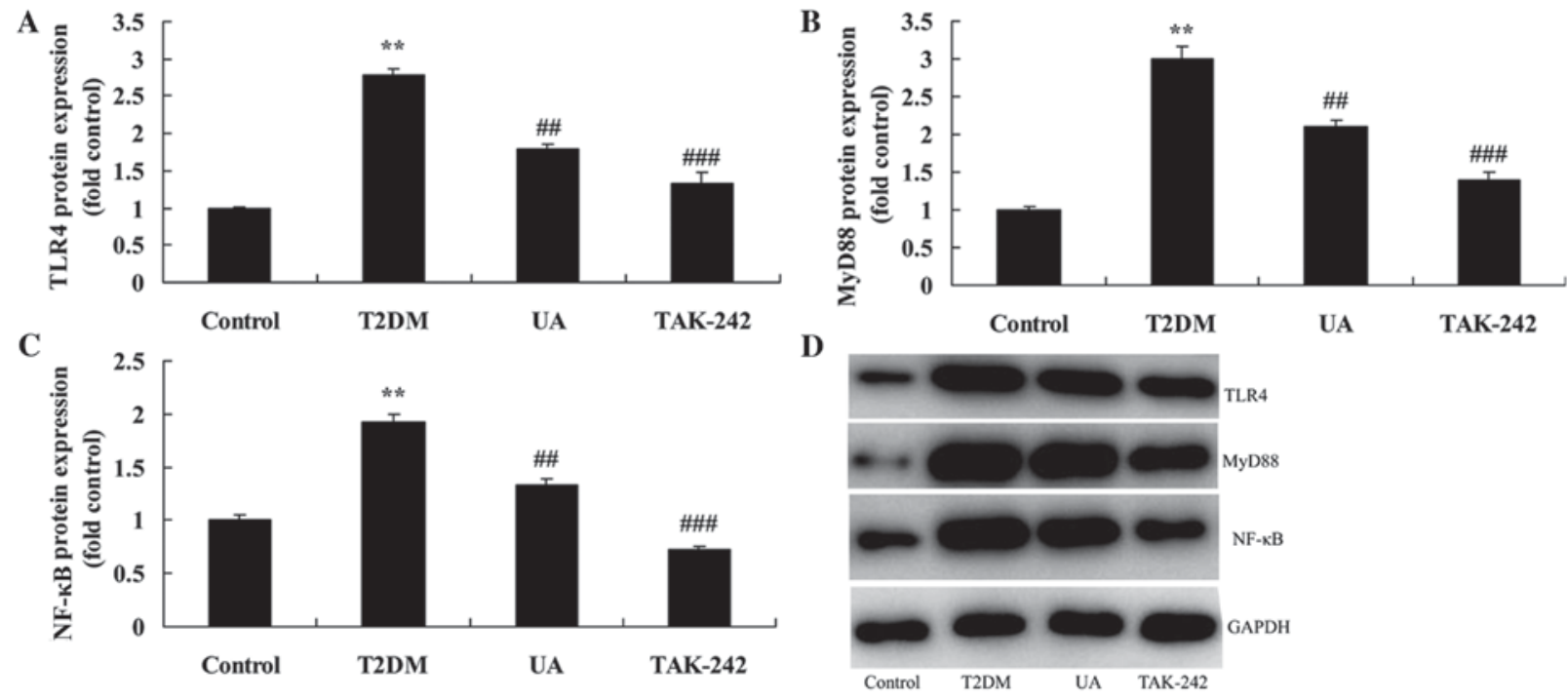

Figure 7. Inhibition of TLR4 increased the anti-inflammation of UA on TLR4 pathway in rat with diabetes-induced nephropathy. (A-C) TLR4, MyD88 and NF- $\kappa$ B protein expression by statistical analysis and (D) western blotting assays. ${ }^{* *} \mathrm{P}<0.01$ compared with control rat group, ${ }^{\# \#} \mathrm{P}<0.01$ compared with diabetes-induced nephropathy group, ${ }^{\# \# \# ~} \mathrm{P}<0.01$ compared with treatment with UA in rat of diabetes-induced nephropathy group. Control, control rat group; T2DM, diabetes-induced nephropathy in rat model group; UA, treatment with UA in rat of diabetes-induced nephropathy group; TAK-242, treatment with UA and TAK-242 in rat of diabetes-induced nephropathy group. UA, ursolic acid; TLR4, Toll-like receptor 4; T2DM, type 2 diabetes mellitus; MyD88, myeloid

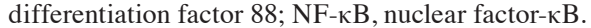

From the view of inflammatory factor expression, the renal tubular epithelial cells HK-2 and macrophages THP-1 are stimulated by TLR4 endogenous and exogenous ligands under hyperglycemia status (10). As a result, expression of inflammatory factors TNF- $\alpha$, MCP-1, IL-6 and IL-8 is remarkably up-regulated within a short time (19). These factors play vitals roles in regulating the innate and adaptive immune response, killing target cells, inducing apoptosis and inducing the production of acute phase reactive protein. Particularly, increased expression of these inflammatory factors have been proved in the serum of DM and DN patients (20). This finding suggest that TLR4 ligands can induce the production of rapid and strong inflammatory response by renal tubular epithelial cells and macrophages under hyperglycemia environment (19). In addition, it demonstrates that renal cell itself can be involved in the inflammatory response of DN through activating TLR4 (20). Zhang et al indicated that UA alleviates early brain injury by suppressing TLR4-mediated inflammatory pathway (12). In the present study, Treatment with UA suppressed TLR4, MyD88 and NF- $\mathrm{kB}$ protein expression in rat with diabetes-induced nephropathy.

Nuclear transcription factor NF- $\mathrm{KB}$ is a transcription factor in eukaryocyte with extensive distribution and function (21). It can be activated by multiple extracellular stimulations. In addition, it can participate in gene regulation under apoptosis and inflammation (22). Meanwhile, it has important physiological and pathological actions (23). Recent study indicated that, NF- $\mathrm{KB}$ is closely related to the genesis and development of DN (23). NF- $\kappa B$ can be activated by numerous physiological and non-physiological stimulations. TLR4 is the major receptor activating NF- $\kappa$ B. Data suggest that NF- $\kappa B$ can be activated by some DM factors, thus it can initiate the transcription of many DN-related genes (24). Moreover, evidence also suggests that NF- $\kappa B$ activation can promote renal interstitial fibrosis through its downstream action (24). Luo et al showed that UA inhibits breast cancer growth via the PI3K/AKT and $\mathrm{NF}-\kappa \mathrm{B}$ signaling pathways (25). Furthermore, we found that the inhibition of TLR4 increased the anti-inflammation of UA on inflammation in rat with diabetes-induced nephropathy through TLR4 pathway.

In conclusion, UA alleviates inflammation and against diabetes-induced nephropathy through TLR4-mediated inflammatory pathway, and UA as a possible therapeutic agent against diabetic nephropathy. UA could be applied in the development of an effective therapeutic strategy for treating diabetes-induced nephropathy.

\section{Acknowledgements}

Not applicable.

\section{Funding}

No funding was received.

\section{Availability of data and materials}

The analyzed data sets generated during the study are available from the corresponding author on reasonable request.

\section{Authors' contributions}

YS designed the present study. JL, NL, SY, ML, BS and YL performed the experiments. YS and JL analyzed the data. YS wrote the manuscript.

\section{Ethics approval and consent to participate}

This study was approved by the Ethics Committee of Chinese PLA General Hospital. 


\section{Patient consent for publication}

Not applicable.

\section{Competing interests}

The authors declare that they have no competing interests.

\section{References}

1. de Boer IH, Sun W, Gao X, Cleary PA, Lachin JM, Molitch ME, Steffes MW and Zinman B; DCCT/EDIC research group: Effect of intensive diabetes treatment on albuminuria in type 1 diabetes: Long-term follow-up of the diabetes control and complications trial and epidemiology of diabetes interventions and complications study. Lancet Diabetes Endocrinol 2: 793-800, 2014.

2. Madsen SM, Thorup AC, Bjerre M and Jeppesen PB: Does 8 weeks of strenuous bicycle exercise improve diabetes-related inflammatory cytokines and free fatty acids in type 2 diabetes patients and individuals at high-risk of metabolic syndrome? Arch Physiol Biochem 121: 129-138, 2015.

3. Aghasi M,Ghazi-ZahediS,Koohdani F, SiassiF,Nasli-EsfahaniE, Keshavarz A, Qorbani M, Khoshamal H, Salari-Moghaddam A and Sotoudeh G: The effects of green cardamom supplementation on blood glucose, lipids profile, oxidative stress, sirtuin-1 and irisin in type 2 diabetic patients: A study protocol for a randomized placebo-controlled clinical trial. BMC Complement Altern Med 18: 18, 2018.

4. Strain WD, Lukashevich V, Kothny W, Hoellinger MJ and Paldánius PM: Individualised treatment targets for elderly patients with type 2 diabetes using vildagliptin add-on or lone therapy (INTERVAL): A 24 week, randomised, double-blind, placebo-controlled study. Lancet 382: 409-416, 2013.

5. Salminen P, Helmiö M, Ovaska J, Juuti A, Leivonen M Peromaa-Haavisto P, Hurme S, Soinio M, Nuutila P and Victorzon M: Effect of laparoscopic sleeve gastrectomy vs laparoscopic Roux-en-Y gastric bypass on weight loss at 5 years among patients with morbid obesity: The SLEEVEPASS randomized clinical trial. JAMA 319: 241-254, 2018.

6. Boss JD, Singh PK, Pandya HK, Tosi J, Kim C, Tewari A, Juzych MS, Abrams GW and Kumar A: Assessment of neurotrophins and inflammatory mediators in vitreous of patients with diabetic retinopathy. Invest Ophthalmol Vis Sci 58: 5594-5603, 2017.

7. Heier M, Margeirsdottir HD, Brunborg C, Hanssen KF, Dahl-Jørgensen K and Seljeflot I: Inflammation in childhood type 1 diabetes; influence of glycemic control. Atherosclerosis 238: 33-37, 2015.

8. On'kin JBKL, Longo-Mbenza B, Tchokonte-Nana V, Okwe AN and Kabangu NK: Hyperbolic relation between beta-cell function and insulin sensitivity for type 2 diabetes mellitus, malaria, influenza, Helicobacter pylori, Chlamydia pneumoniae, and hepatitis $C$ virus infection-induced inflammation/oxidative stress and temporary insulin resistance in Central Africans. Turk J Med Sci 47: 1834-1841, 2017

9. Doody NE, Dowejko MM, Akam EC, Cox NJ, Bhatti JS, Singh P and Mastana SS: The role of TLR4, TNF- $\alpha$ and IL- $1 \beta$ in type 2 diabetes mellitus development within a North Indian population. Ann Hum Genet 81: 141-146, 2017.

10. Perry BD, Rahnert JA, Xie Y, Zheng B, Woodworth-Hobbs ME and Price SR: Palmitate-induced ER stress and inhibition of protein synthesis in cultured myotubes does not require Toll-like receptor 4. PLoS One 13: e0191313, 2018.
11. Yang S, Zhang J, Wang S, Shi J and Zhao X: Knockdown of angiopoietin-like protein 2 ameliorates diabetic nephropathy by inhibiting TLR4. Cell Physiol Biochem 43: 685-696, 2017.

12. Zhang T, Su J, Guo B, Zhu T, Wang K and Li X: Ursolic acid alleviates early brain injury after experimental subarachnoid hemorrhage by suppressing TLR4-mediated inflammatory pathway. Int Immunopharmacol 23: 585-591, 2014.

13. Zhai M, Guo J, Ma H, Shi W, Jou D, Yan D, Liu T, Tao J, Duan J, Wang Y, et al: Ursolic acid prevents angiotensin II-induced abdominal aortic aneurysm in apolipoprotein E-knockout mice. Atherosclerosis 271: 128-135, 2018.

14. Zhou W, Lin L, Cheng Y and Liu Y: Ursolic acid improves liver transplantation and inhibits apoptosis in miniature pigs using donation after cardiac death. Cell Physiol Biochem 43: 331-338, 2017.

15. Yu EY, Wong CK, Ho SY, Wong SY and Lam CL: Can HbA1c replace OGTT for the diagnosis of diabetes mellitus among Chinese patients with impaired fasting glucose? Fam Pract 32: 631-638, 2015.

16. Yang YJ, Wu CT, Ou HY, Lin $\mathrm{CH}$, Cheng $\mathrm{HC}$, Chang WL, Chen WY, Yang HB, Lu CC and Sheu BS: Male non-insulin users with type 2 diabetes mellitus are predisposed to gastric corpus-predominant inflammation after $H$. pylori infection. J Biomed Sci 24: 82, 2017.

17. Feng Y, Jiang CD, Chang AM, Shi Y, Gao J, Zhu L and Zhang Z: Interactions among insulin resistance, inflammation factors, obesity-related gene polymorphisms, environmental risk factors, and diet in the development of gestational diabetes mellitus. J Matern Fetal Neonatal Med: 1-9, 2018.

18. Wang XT, Gong Y, Zhou B, Yang JJ, Cheng Y, Zhao JG and Qi MY: Ursolic acid ameliorates oxidative stress, inflammation and fibrosis in diabetic cardiomyopathy rats. Biomed Pharmacother 97: 1461-1467, 2018.

19. Chen G, Sun W, Liang Y, Chen T, Guo W and Tian W: Maternal diabetes modulates offspring cell proliferation and apoptosis during odontogenesis via the TLR4/NF- $\kappa \mathrm{B}$ signalling pathway. Cell Prolif 50, 2017.

20. Li C, Che LH, Ji TF, Shi L and Yu JL: Effects of the TLR4 signaling pathway on apoptosis of neuronal cells in diabetes mellitus complicated with cerebral infarction in a rat model. Sci Rep 7: 43834, 2017.

21. Tang ST, Su H, Zhang Q, Tang HQ, Wang CJ, Zhou Q, Wei W, Zhu HQ and Wang Y: Sitagliptin inhibits endothelin-1 expression in the aortic endothelium of rats with streptozotocin-induced diabetes by suppressing the nuclear factor- $\kappa \mathrm{B} / \mathrm{I} \kappa \mathrm{B} \alpha$ system through the activation of AMP-activated protein kinase. Int $\mathrm{J}$ Mol Med 37: 1558-1566, 2016.

22. Ge Y, Paisie TK, Newman JRB, McIntyre LM and Concannon P. UBASH3A mediates risk for type 1 diabetes through inhibition of T-cell receptor-induced NF- $\mathrm{KB}$ signaling. Diabetes 66: 2033-2043, 2017.

23. Guo C, Zhang L, Nie L, Zhang N, Xiao D, Ye X, Ou M, Liu Y, Zhang B, Wang M, et al: Association of polymorphisms in the MyD88, IRAK4 and TRAF6 genes and susceptibility to type 2 diabetes mellitus and diabetic nephropathy in a southern Han Chinese population. Mol Cell Endocrinol 429: 114-119, 2016.

24. Wang X, Li D, Fan L, Xiao Q, Zuo H and Li Z: CAPE-pNO2 ameliorated diabetic nephropathy through regulating the Akt/NF- $\kappa$ B/iNOS pathway in STZ-induced diabetic mice. Oncotarget 8: 114506-114525, 2017.

25. Luo J, Hu YL and Wang H: Ursolic acid inhibits breast cancer growth by inhibiting proliferation, inducing autophagy and apoptosis, and suppressing inflammatory responses via the PI3K/ $\mathrm{AKT}$ and NF- $\mathrm{kB}$ signaling pathways in vitro. Exp Ther Med 14: 3623-3631, 2017. 